\title{
Branch Thinning and the Large-Scale, Self-Similar Structure of Trees
}

\author{
Lars Hellström, ${ }^{1,2}$ Linus Carlsson, ${ }^{1,2}$ Daniel S. Falster, ${ }^{3,4}$ Mark Westoby, ${ }^{4}$ and Åke Brännströmm ${ }^{1,5, *}$ \\ 1. Department of Mathematics and Mathematical Statistics, Umeå University, SE-90187 Umeå, Sweden; 2. School of Education, \\ Culture and Communication, Mälardalen University, SE-721 23 Västerås, Sweden; 3. Evolution and Ecology Research Centre, and \\ School of Biological, Earth and Environmental Sciences, University of New South Wales, Sydney, New South Wales 2052, Australia; \\ 4. Department of Biological Sciences, Macquarie University, Sydney, New South Wales 2109, Australia; 5. Evolution and Ecology Program, \\ International Institute for Applied Systems Analysis, A-2361 Laxenburg, Austria
}

Submitted November 7, 2016; Accepted November 21, 2017; Electronically published April 10, 2018

Online enhancements: appendix.

\begin{abstract}
Branch formation in trees has an inherent tendency toward exponential growth, but exponential growth in the number of branches cannot continue indefinitely. It has been suggested that trees balance this tendency toward expansion by also losing branches grown in previous growth cycles. Here, we present a model for branch formation and branch loss during ontogeny that builds on the phenomenological assumption of a branch carrying capacity. The model allows us to derive approximate analytical expressions for the number of tips on a branch, the distribution of growth modules within a branch, and the rate and size distribution of tree wood litter produced. Although limited availability of data makes empirical corroboration challenging, we show that our model can fit field observations of red maple (Acer rubrum) and note that the age distribution of discarded branches predicted by our model is qualitatively similar to an empirically observed distribution of dead and abscised branches of balsam poplar (Populus balsamifera). By showing how a simple phenomenological assumption - that the number of branches a tree can maintain is limited-leads directly to predictions on branching structure and the rate and size distribution of branch loss, these results potentially enable more explicit modeling of woody tissues in ecosystems worldwide, with implications for the buildup of flammable fuel, nutrient cycling, and understanding of plant growth.
\end{abstract}

Keywords: branching structure, self-similarity, tree architecture, wood litter.

\section{Introduction}

Many plant species exhibit self-similarity, in that they appear geometrically, topologically, or statistically similar when viewed at different scales (Ferraro et al. 2005; Prusinkiewicz

* Corresponding author; e-mail: ake.brannstrom@math.umu.se.

ORCIDs: Hellström, http://orcid.org/0000-0001-6140-180X; Falster, http://orcid .org/0000-0002-9814-092X; Westoby, http://orcid.org/0000-0001-7690-4530; Brännström, http://orcid.org/0000-0001-9862-816X.

Am. Nat. 2018. Vol. 192, pp. E000-E000. (C) 2018 by The University of Chicago. 0003-0147/2018/19201-57367\$15.00. All rights reserved.

DOI: $10.1086 / 697429$ et al. 2007; Godin and Ferraro 2010). Such self-similarity underlies the stunning computer-generated images of plants on display in Prusinkiewicz and Lindenmayer's (1990) influential book The Algorithmic Beauty of Plants, as well as the metabolic theory of ecology (West et al. 1997; Enquist et al. 1999). Reflecting its importance, several different concepts of self-similarity have been developed and used to model and understand plant form (Prusinkiewicz 2004; Godin and Ferraro 2010). For many tree species, however, it is not immediately obvious how self-similarity is maintained during botanical growth, because the trivial way of maintaining self-similarity under growth - to uniformly scale everything up - is inconsistent with the nature of woody plants. Heartwood is inert, so once it is formed the heartwood section of a given growth module cannot increase in length, only in girth, whereas branches grow in length by adding new growth modules at the tips.

To understand how self-similarity is maintained as trees grow, it may help to consider not only the growth of new shoots but also how branches are discontinued and lost. Death and self-pruning of branches is in fact common in many species (Schaffner and Tyler 1901; Dewit and Reid 1992; Heräjärvi 2001; Mäkinen and Hein 2006); factors that influence this process have been thoroughly studied, as high density of branches is generally considered negative in silviculture (Mäkinen 1999; Mäkinen and Colin 1999; Ford 2014). Branch thinning is a less obvious process in the transformation of a tree than the growth of new shoots, in that it may seem random and any single event could easily be attributed to damage by external forces, but by considering changes over a span of several growth cycles (fig. 1) it is possible to see that some manner of branch thinning must be part of the normal development of many tree species. Figure 2 shows specimens of Scots pine and Blue Mountains ash that have lost branches during ontogeny. 


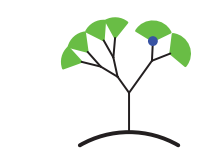

$5 \mathrm{GC}$

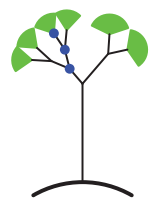

8 GC

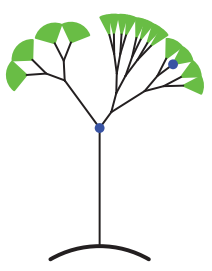

$11 \mathrm{GC}$

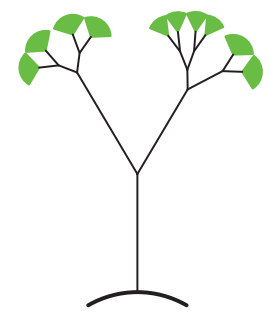

$14 \mathrm{GC}$

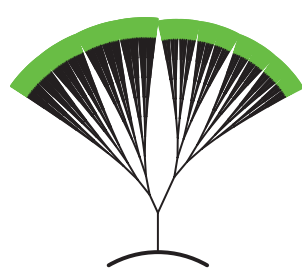

$11 \mathrm{GC}$

with thinning

without

thinning

Figure 1: Branch thinning is part of the normal development of many tree species. The four leftmost panels conceptually show the development of a tree with branch thinning: many side branches are discontinued, and as a result the distance between adjacent branching points increases. Branching points that remain in the next panel are marked with a dot. By contrast, the last panel shows the result of pure primary growth without branch thinning: the exponential increase in the number of branches forces them to be so densely packed that they overlap even when drawn with thin lines.

While branch thinning has been investigated both empirically (Dewit and Reid 1992; Mäkinen 1999) and theoretically (Mäkelä 1997; Eschenbach 2005), no quantitative model of branch thinning has as yet been developed and applied to make predictions for both branching architecture at a point in time and production over time of a size mixture of dead and abscised branches. Functional-structural plant models such as the GreenLab model (Cournède et al. 2008) should in principle be capable of making such predictions, though except for Eschenbach (2005) they have not to our knowledge been applied for this purpose, and their predictions would likely need to be derived numerically through computer simulation. Here, we introduce and analyze an elementary model of branch thinning that rests on the assumption that the number of tips on a branch increases to, but does not exceed, a carrying capacity that depends on the branch age. We show how this model allows both quantitative and qualitative predictions of branching architecture at specific times and production of a size mixture of dead and abscised branches over time.

\section{Methods}

We consider a model of tree growth in which a tree develops over time by following two basic rules. First, in each growth cycle a growth module is added at each tip, and at the distal end of that growth module some average number $(\mu \geq 1)$ of tips is formed. Second, if a branch that is $n$ growth cycles old exceeds a carrying capacity of $K(n)$ tips, it may discard one or several subbranches in a process of branch thinning. We quantify the resultant branching structure, as illustrated in figure 3, by counting the number of growth modules $g(\ell, n)$ at a distance $\ell$ growth cycles from the start of the branch under consideration. Here, we use "distance" to mean that the growth modules are formed $\ell$ growth cycles after the tree started growing and they are thus $n-\ell$ growth cycles old, but by assuming that all growth modules have roughly equal length we can also consider this an actual distance. Table 1 gives an overview of the parameters and statistical quantities that we use in our derivations.

To complete the model specification, we need to specify the form of the branch carrying capacity, the details of how a tip branches into an average $\mu>1$ more tips, and the details of how subbranches are discarded once a branch exceeds its carrying capacity. We assume that the branch carrying capacity is given by

$$
K(n)=\alpha(n+1)^{d},
$$

which is a simple, increasing function in $n$ that for $d=3$ is compatible with the hypothesis of spatial constraints as the main limiting factor.

As we show in Results, the assumptions above suffice for deriving approximate analytical expressions for the largescale branching structure of trees, without specifying the details of the branching process or how subbranches are 
a

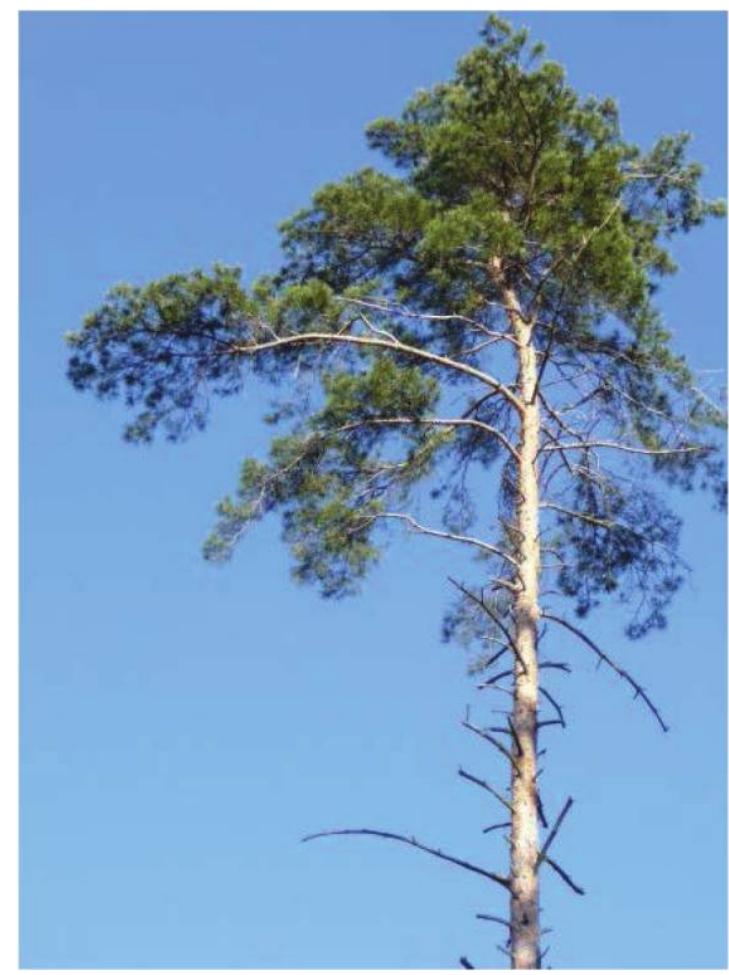

b

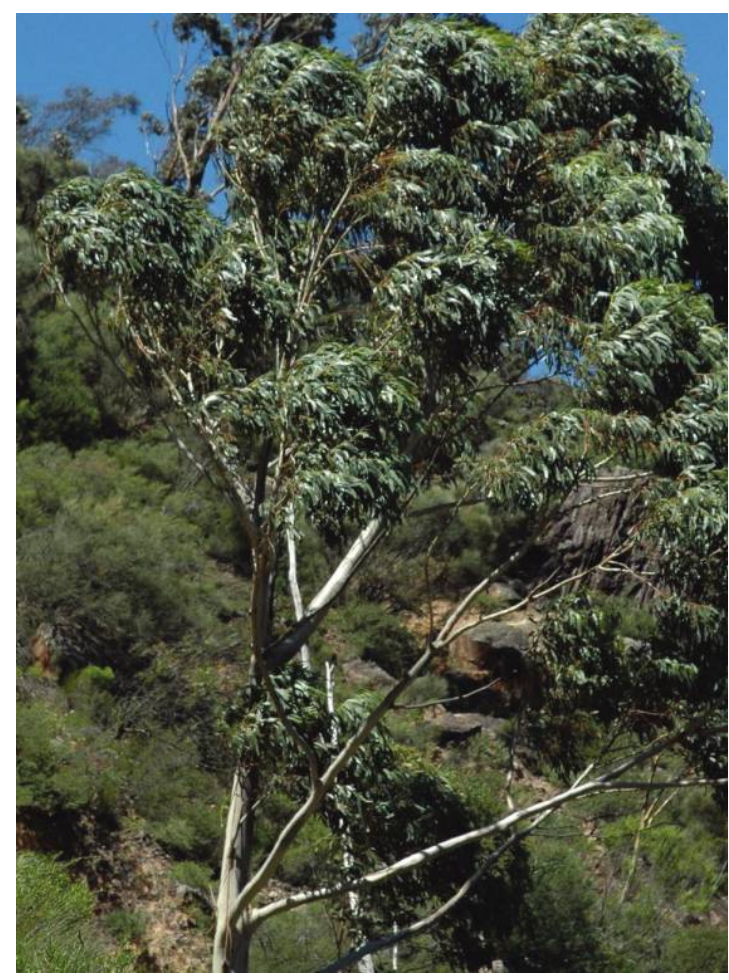

Figure 2: Many tree species lose a significant number of branches through ontogeny. $a$, Scots pine, Pinus sylvestris, with stumps of dead branches on the stem, indicating larger branches that have been lost. $b$, Blue Mountains ash, Eucalyptus oreades, without visible stumps of dead branches but that must nonetheless have lost branches during ontogeny. In particular, when the now large and sturdy branches were first formed they held smaller branches near to the main stem that were later lost.

discarded. To illustrate and corroborate our analytical conclusions, we use an individual-based simulation described in appendix section Details of the Individual-Based Simulation (the appendix is available online) and presented in figure 4; the main idea is to alternate between growth steps (working as in an L-system; Lindenmayer 1968; Prusinkiewicz and Lindenmayer 1990) and thinning steps, where subbranches are discarded from a branch with probability $p$ when the branch exceeds its carrying capacity. To test the robustness of our conclusions, we also implement more realistic assumptions and show in appendix section Robustness of Results that these assumptions do not alter our findings. Source code and data used in this article are available at Zenodo: https://zenodo.org/record/1160465 (Hellström 2018). ${ }^{1}$

\section{Results}

Based on our model assumption of a branch carrying capacity, we derive approximate analytical predictions for

1. Code that appears in the American Naturalist is provided as a convenience to the readers. It has not necessarily been tested as part of the peer review. the large-scale branching structure of our modeled trees. These include the number of tips on a branch, the distribution of growth modules within a branch, the rate of wood litter production during ontogeny, and the size distribution of discarded branches. Finally, we fit our model predictions to observations of red maple (Acer rubrum) and qualitatively compare our calculated size distribution of discarded branches to observations of balsam poplar (Populus balsamifera).

\section{The Number of Tips on a Branch}

An immediate conclusion from combining the fixed branching ratio $\mu$ with the branch carrying capacity $K(n)$ is that the expected number of tips $b(n)$ on a branch of age- $n$ growth cycles must fulfill

$$
b(n) \leq \min \left\{\mu^{n}, K(n)\right\},
$$

since primary growth leads to an increase in the number of tips by a factor $\mu$, whereas branch thinning reduces that number when it exceeds the $K(n)$ saturation bound. The equilibrium between these two opposing processes is a 


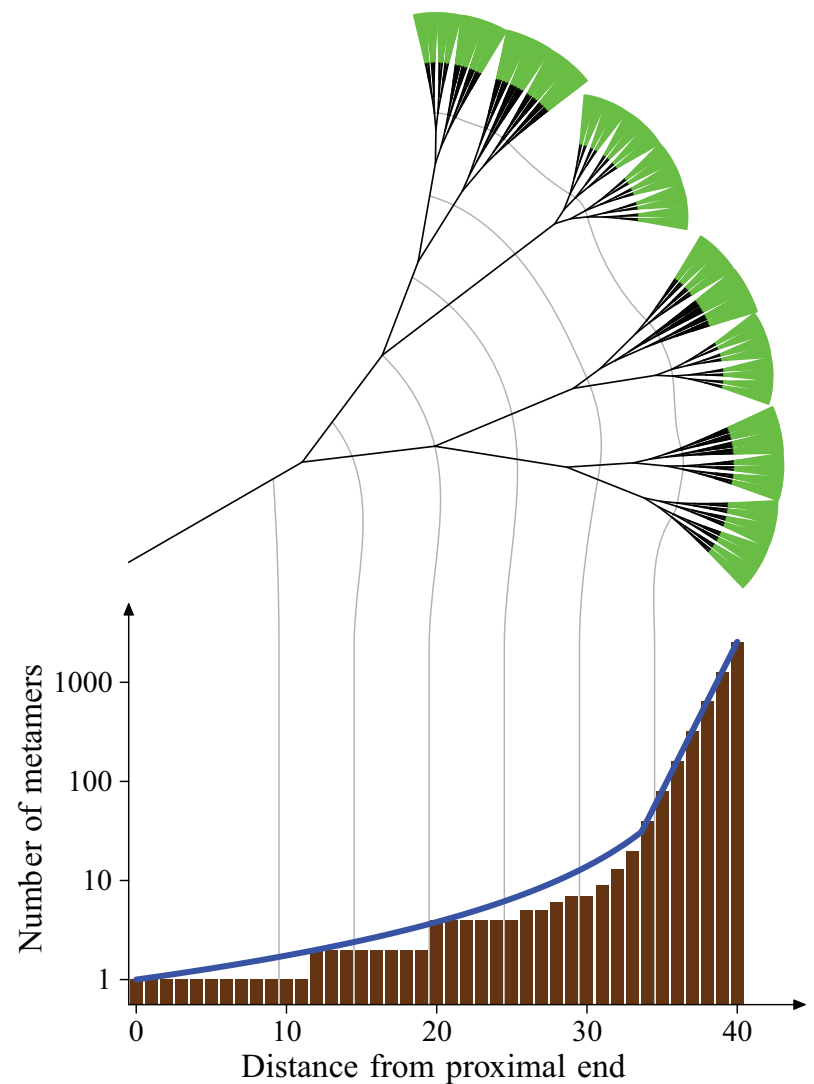

Figure 3: We quantify branching patterns by counting the number of growth modules at a given distance $\ell$ from the proximal end of a branch or a tree, measured in growth cycles. The top image shows the structure of a simulated branch, produced by alternating growth and branch thinning for 40 growth cycles. The histogram shows the number of growth modules at each distance. The curve in the graph shows the theoretical expectation $g(\ell, n)$ of equation (7). Parameters: $\mu=2, \alpha=3, d=2, n=40$, and $\beta=1.5229$ (chosen to make $g(n, n)$ match the number of tips in the branch). See table 1 for variable and parameter definitions.

branch whose number of tips is just below the carrying capacity. For small branches, however, the exponential growth $\mu^{n}$ has not yet reached the branch carrying capacity, and for these branches $\mu^{n}$ constitutes the practical upper bound.

If the branch-thinning process removes at most a fixed proportion $c$ of all tips of the tree, we would also have the lower bound

$$
b(n) \geq \min \left\{\mu^{n}, c K(n)\right\} .
$$

Hence, with exception of an early phase with exponential growth, the expected number of tips on a branch will lie between $c \alpha(n+1)^{d}$ and $\alpha(n+1)^{d}$, as $K(n)=\alpha(n+1)^{d}$. In deriving analytical results, we will assume that

$$
b(n)=\min \left\{\mu^{n}, \beta(n+1)^{d}\right\} .
$$

This assumption (i.e., that $\beta$ is a fixed value between $c \alpha$ and $\alpha$, which simplifies arguments and presentation of results) is in good agreement with our individual-based simulations and robust under an alternative process of branch thinning (see appendix section Robustness of Results). Most of our results can be derived without this assumption but then take the form of an inequality in which the predicted quantity is bounded from below and above using equations (2) and (3), respectively. Note that equation (4) assumes immediate branch thinning when a branch first exceeds its branch carrying capacity. To model gradual transitions, a smooth approximation of the minimum function can be used. We explore this possibility in Empirical Corroboration.

As a first result, it follows from equation (4) that the distances between two consecutive branching points are (on average) first constant near the tree periphery and then increase geometrically as the tree is traversed from the distal end to the proximal end. Because $b(n)$ in the self-thinning region follows a power law, its value will on average double between two consecutive branching points when one traverses a branch from the distal (tip) to the proximal end (stem), since a branching point joins two subbranches that on average have equal size. Hence, $n$ will have to grow by a factor roughly $2^{1 / d}$ between branching points, depending on the exponent $d$ but not on the position $n$.

\section{The Distribution of Growth Modules within a Branch}

We quantify tree branching structure, as illustrated in figure 3 , by counting the number of growth modules $g(\ell, n)$

\begin{tabular}{|c|c|c|}
\hline Symbol & Meaning & Uni \\
\hline$\alpha$ & $\begin{array}{l}\text { Proportionality constant in branch carrying } \\
\text { capacity }\end{array}$ & \\
\hline$\beta$ & $\begin{array}{l}\text { Realized proportionality constant in statistical } \\
\text { quantities }\end{array}$ & \\
\hline$d$ & Exponent in the branch carrying capacity & \\
\hline$\mu$ & $\begin{array}{l}\text { Average number of tips formed at a growth } \\
\text { module }\end{array}$ & \\
\hline$M_{n}$ & $\begin{array}{l}\text { Biomass of a growth module } n \text { growth cycles } \\
\text { old }\end{array}$ & \\
\hline$p$ & $\begin{array}{l}\text { Pruning probability in our individual-based } \\
\text { model }\end{array}$ & \\
\hline$b(n)$ & $\begin{array}{l}\text { Average number of tips on a branch } n \text { growth } \\
\text { cycles old }\end{array}$ & \\
\hline$g(\ell, n)$ & $\begin{array}{l}\text { Average number of } \ell \text { th growth cycle descen- } \\
\text { dants of a growth module } n \text { growth cycles old }\end{array}$ & \\
\hline$K(n)$ & $\begin{array}{l}\text { Maximum sustainable number of tips on a } \\
\text { branch } n \text { growth cycles old, also referred to } \\
\text { as the branch carrying capacity }\end{array}$ & \\
\hline
\end{tabular}

Table 1: Parameters and central statistical quantities

Note: A dash symbol indicates that the value is dimensionless. 


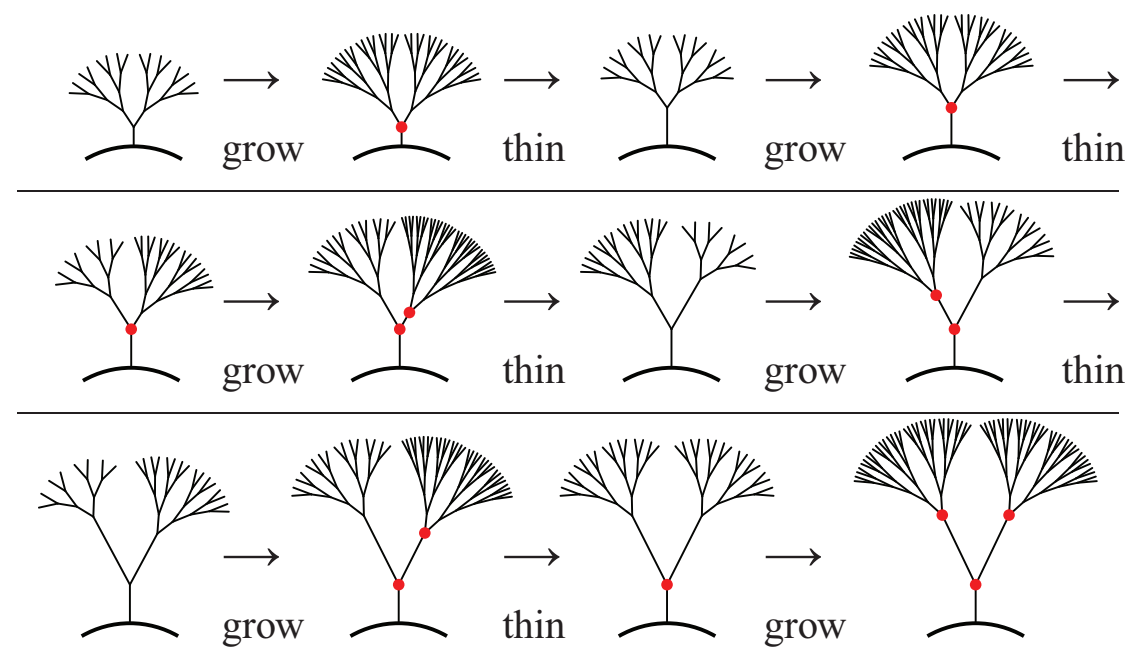

Figure 4: Illustration of the individual-based model. Each image represents one stage in a branch growth simulation, shown alternatingly before growth and before branch thinning. Dots indicate branches that exceed their carrying capacity. Note that, due to the probabilistic nature of our individual-based model, some branches may remain above their carrying capacity also after branch thinning. Parameters: $\alpha=3$, $d=2, \mu=2$, and $p=10 \%$. See table 1 for variable and parameter definitions.

at a distance $\ell$ growth cycles from the root of a tree of age- $n$ growth cycles. By definition, $g(0, n)=1$ and $g(n, n)=$ $b(n)$. Assuming that any two branches of the same age can be considered to be independent and to have the same statistical properties, we show in appendix section Identities for the Mean and Variance that $g(\ell, n)$ is uniquely determined by the function $b$ and equal to

$$
g(\ell, n)=\frac{b(n)}{b(n-\ell)}
$$

The argument is that the full tree has $b(n)$ tips, whereas if one traverses $\ell$ growth cycles from the root toward a tip, then one reaches a branch of age $n-\ell$ growth cycles with on average $b(n-\ell)$ tips. The expected number of such branches is by definition $g(\ell, n)$, so by bookkeeping $b(n)=g(\ell, n) b(n-\ell)$, which leads to equation (5). Individual-based simulations confirm that a process of alternating primary growth and branch thinning does indeed give this branching structure, as shown in figure 5 .

A direct consequence of equation (5) is that the expected number of branches produced by a growth module that is a $\ell$ th growth cycles descendant of a branch of age- $n$ growth cycles equals the quotient

$\frac{\text { number of }(\ell+1) \text { th growth cycle growth modules }}{\text { number of } \ell \text { th growth cycle growth modules }}=\frac{g(\ell+1, n)}{g(\ell, n)}$

$$
=\frac{b(n) / b(n-\ell-1)}{b(n) / b(n-\ell)}=\frac{b(n-\ell)}{b(n-\ell-1)}=g(1, n-\ell) .
$$

The right-hand side is the expected number of subbranches of a tree of age $n-\ell$, thus implying uniformity in that a subbranch of a tree has the same average branching structure as a tree that has existed for the same number of growth cycles.

From combining equations (4) and (5) and restricting attention to the branch-thinning region, one arrives at an equation for the number of growth modules:

$$
g(\ell, n)=\frac{b(n)}{b(n-\ell)}=\frac{\beta(n+1)^{d}}{\beta(n-\ell+1)^{d}}=\left(1-\frac{\ell}{n+1}\right)^{-d},
$$

which is interesting in that the only parameter remaining is the carrying-capacity exponent $d$. Equation (7) leads to two salient predictions.

First, the branching structure is self-similar, in the sense that on average a large branch looks like a scaled-up version of a small branch when we compare the branch-thinning regions. More precisely, for a constant ratio of $\ell$ to $n+1$ one gets the same expected value $g(\ell, n)$ of $\ell$ th growth cycle growth modules regardless of the overall branch age $n$. Figure 6 illustrates this for two simulated individuals, by counting growth modules at the proximal-distal midpoint of two branches of different age: the number is the same in both cases. Figure A2 (figs. A1-A5 are available online) shows a plot of $\ell /(n+1)$ against $g(\ell, n)$ for three different values of $n$; the three curves diverge only when $b(n-\ell)$ switches over to $\mu^{n-\ell}$ (the exponential-growth region). See table 1 for variable and parameter definitions.

Second, after rescaling, the branching structure depends chiefly on $d$; other parameters have little or no influence 


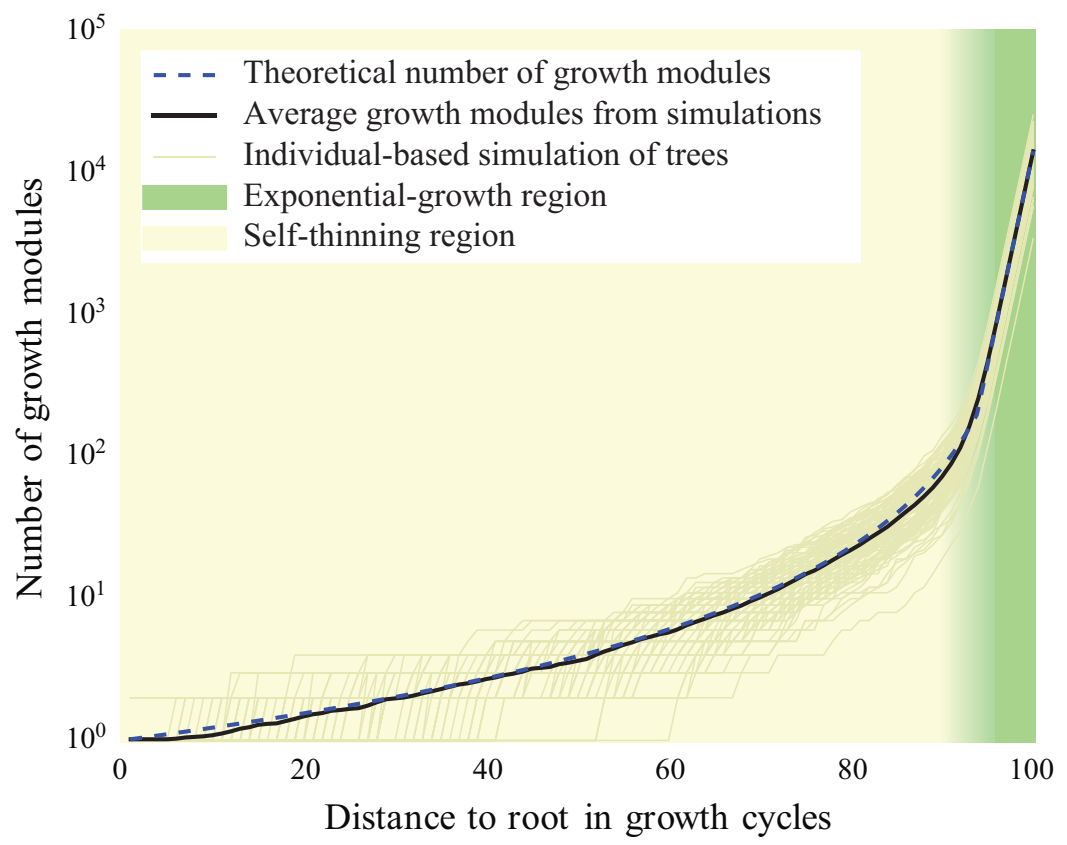

Figure 5: Individual-based simulations corroborate equation (5), the theoretical number of growth modules $g(\ell, n)$ depicted in figure 3 . The spread in simulated trees due to randomness in the process is several times larger than the difference between simulation average and theoretically predicted average. Parameters are $\mu=2, \alpha=3, \beta=0.5, d=2, p=10 \%$, and $n=100$. See table 1 for variable and parameter definitions.

on this. For $d=1$, one expects $g(\ell, n)=2$ (first branching has taken place) at $\ell /(n+1)=0.5$, whereas for $d=2$ one expects the first branching at $\ell /(n+1) \approx 0.29$ and expects $g(\ell, n)=4$ at $\ell /(n+1)=0.5$.
In appendix section Empirical Measurement of Model Parameters, we discuss data requirements and statistical procedures for empirically estimating the parameters of equations (4) and (7).

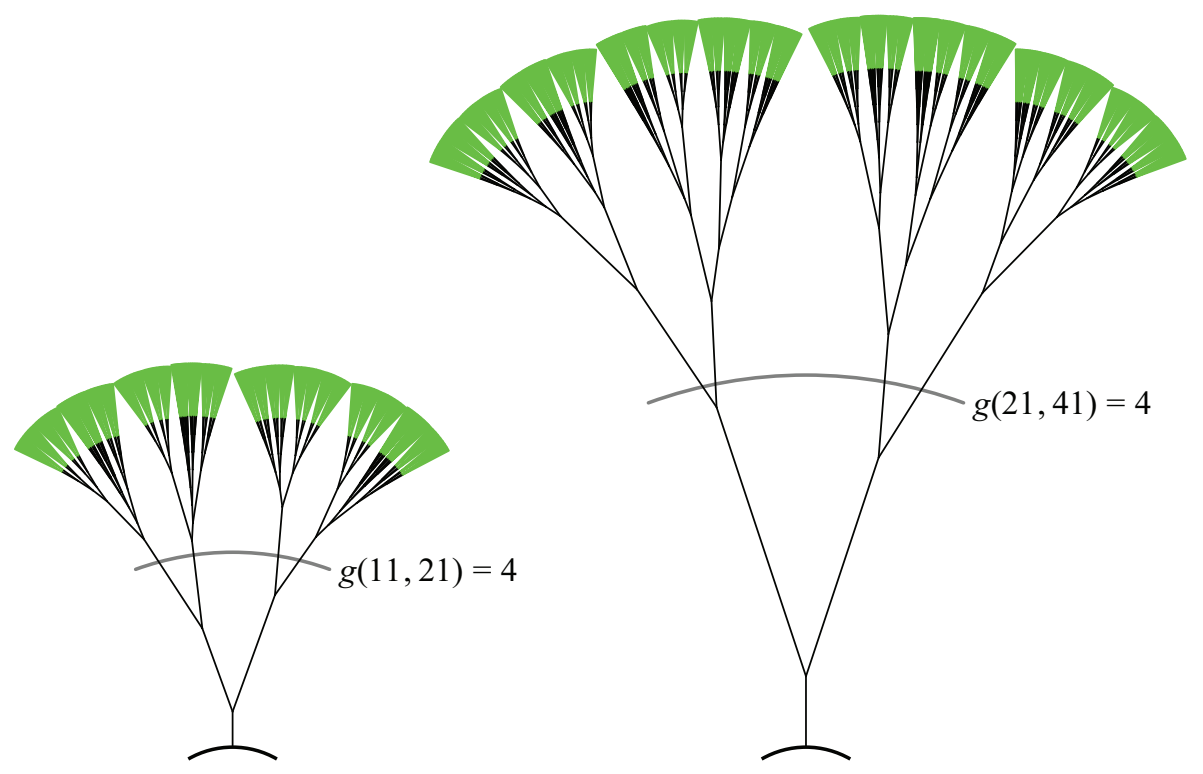

Figure 6: Emergent branching structure is self-similar; two simulated individuals of different sizes still branch at roughly the same rate, relative to the full size of the branch being considered. The gray lines mark the proximal-distal midpoint of the branches, and both intersect the same number of growth modules (namely, four). Parameters are $\mu=2, \alpha=3$, and $d=2$. 


\section{Wood Litter Production during Growth}

The number of growth modules at a distance $\ell$ growth cycles from the root of a tree $n$ growth cycles old (eq. [5]) decreases with $n$ as these growth modules gradually disappear because of branch thinning, whereas new ones form only from tips. The difference $g(\ell, n)-g(\ell, n+1)$ is thus a measure of the expected number of growth modules of age $n-\ell$ that are lost in a branch between growth cycles $n$ and $n+1$ of that branch. Importantly, some of these growth modules are lost because they are part of a larger branch that is discarded. To determine the expected number of branches of a given age that are lost in a growth cycle, we subtract the expected number of growth modules aged $n-\ell$ that are part of larger branches being lost. Using equation (5), this gives

$$
\begin{aligned}
(g(\ell, n)-g(\ell, n+1)) & -g(1, n-\ell+1)(g(\ell-1, n) \\
& -g(\ell-1, n+1)) \\
= & g(\ell, n+1)\left(\frac{b(n-\ell+1)^{2}}{b(n-\ell) b(n-\ell+2)}-1\right)
\end{aligned}
$$

as the expected number of branches of age $-n-\ell$ growth cycles that are discarded due to branch thinning directly at their supplying growth module. The corresponding age distribution of discarded branches, shown in figure A3, has a peak at the age at which branches hit their carrying capacity and an extended tail of older branches.
In addition to describing the age and number of branches that are on average lost during growth, we can determine the wood litter production measured as the biomass lost. If a growth module that is $n$ growth cycles old has mass $M_{n}$, the expected wood litter production from a tree of age $n$ during the following growth cycle becomes

$$
\sum_{\ell=0}^{n}(g(\ell, n)-g(\ell, n+1)) M_{n-\ell} .
$$

We approximate $M_{n}$ using allometric relationships. McMahon and Kronauer (1976) conclude that the branch radius $r$ grows as branch length to the power 3/2; thus, $n^{3 / 2} \propto r$ and $M_{n} \propto r^{2}$, which combine to $M_{n} \propto r^{2} \propto n^{3}$. Hence, the expected wood litter of age- $n-\ell$ growth modules from a branch of age $n$ over one growth cycle is

$$
\begin{aligned}
(g(\ell, n) & -g(\ell, n+1)) M_{n-\ell} \\
& \propto\left(\frac{b(n)}{b(n-\ell)}-\frac{b(n+1)}{b(n+1-\ell)}\right)(n-\ell)^{3} .
\end{aligned}
$$

Figure 7 shows that, also by biomass, there is a peak in wood litter of branches that have just reached their carrying capacity, regardless of total tree age. In wood litter produced by older trees, there is also a significant fraction of large-diameter growth modules. For the latter fraction, there are two opposing trends in their contribution to the distribution of wood litter biomass: on the one hand, a decrease in thinning frequency, and on the other hand, an increase in volume of individual growth modules.

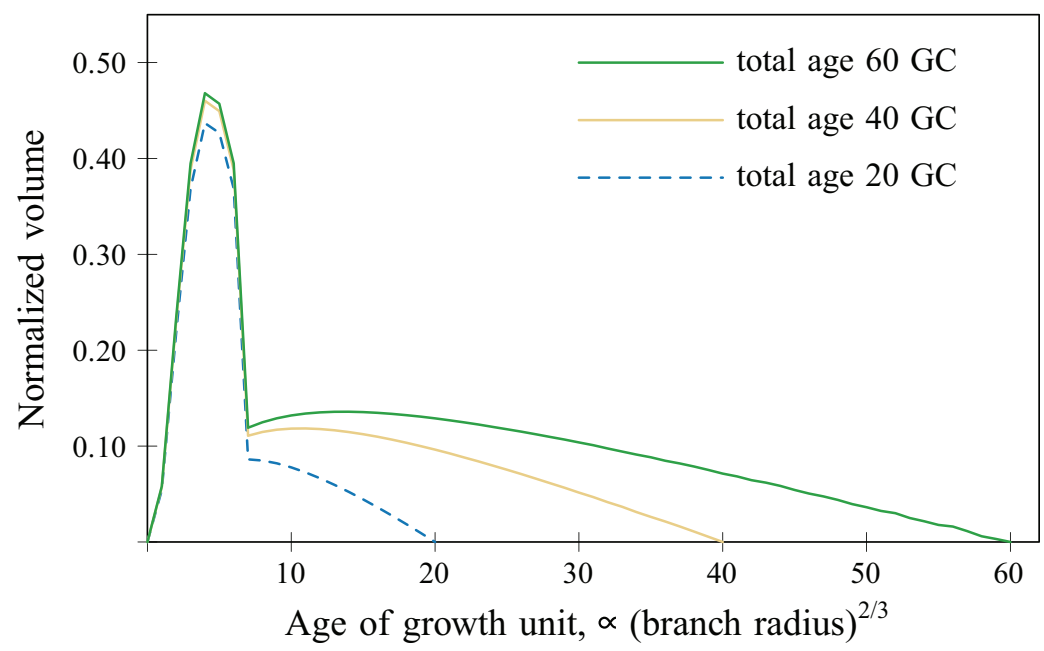

Figure 7: By volume, the distribution of wood litter has a peak around the transition from the exponential-growth region to the branchthinning region, but for older branches the fraction of large-diameter material increases. The normalization of wood volume is done in two steps. First, the volume of one age-10 growth module is taken as the volume unit. Second, the numbers obtained are divided by the expected number of age-10 growth modules to make the distributions for different age branches comparable. Parameters: $\beta=2$, $d=2$, and $\mu=2$. See table 1 for variable and parameter definitions. 


\section{Empirical Corroboration}

Wilson (1966, fig. 10) provides data for red maple (A. rubrum) that correspond directly to our $b(n)$ function: how the number of "long shoots" in a tree varies on average with the number of "groups." A group here is the analog of a whorl in pine trees, so the number of groups on the stem corresponds to the age of the tree in growth cycles. We fit equation (4) using nonlinear regression to the 29 groups. Assuming an offset of four growth cycles, the best model fit of $b(n)$, equation (4), based on minimizing the sum of squared errors with the data on a logarithmic scale, gives parameters $\beta=6.69, d=1.44$, and $\mu=1.51$, with a coefficient of variation $R^{2}=0.9834$ on the original linear scale, thus explaining more than $98 \%$ of the variation in the data. Figure 8 shows the estimated $b(n)$ plotted against the data on a semilogarithmic scale. Wilson $(1966,6)$ remarks that old groups are being lost, so high group numbers are systematically underestimating the true age of the tree; a similar regression using only the first 25 groups gives $R^{2}=0.9972$ (fig. A4).

Wilson's data are qualitatively similar to our expression $b(n)$, as shown in figure 8 , with a near-linear section for low group numbers (the exponential-growth region) and a sharp

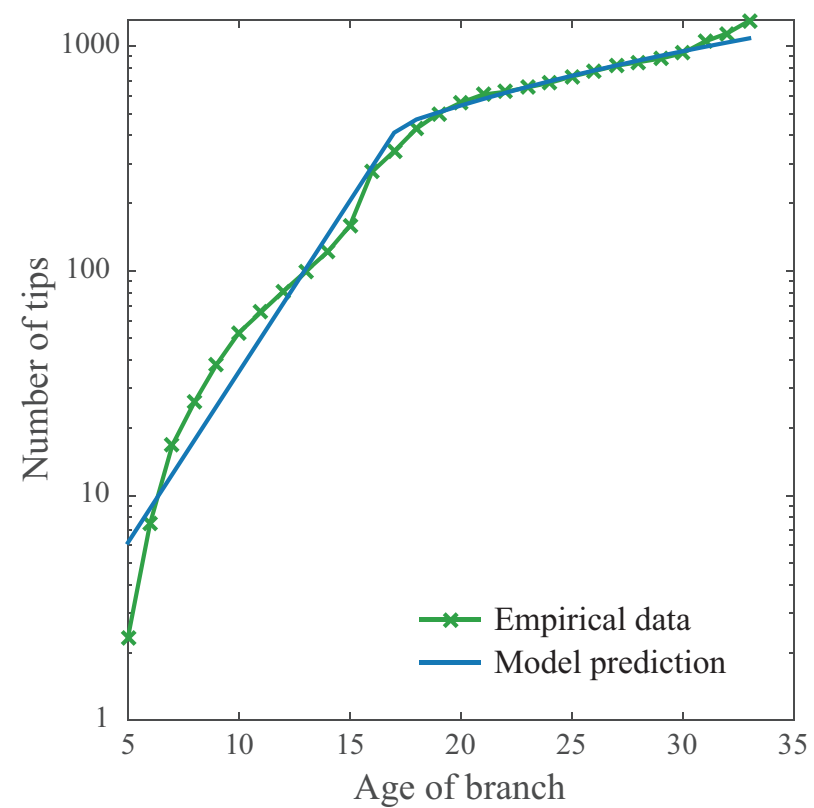

Figure 8: Our prediction for the number of tips compares favorably with empirical measurements. The function $b(n)$ defined by equation (4) is fitted to empirical data from Wilson (1966) by minimizing the sum of square errors with the data on a logarithmic scale. The estimated parameters are $\beta=6.69, d=1.44$, and $\mu=1.42$, with $R^{2}=0.9834$ on the original linear scale. See table 1 for variable and parameter definitions. bend leading to much slower growth at high group numbers (the branch-thinning region). The transition seems to happen at around $n=15$, which is notably higher than we see in our individual-based simulations, but the branch structure of red maple - with the two separate classes of short- and long-shoot branches, where the former carry leaves but no smaller branches-makes it likely that the $\beta$ parameter could be fairly high, and this in turn should lead to a large exponential-growth region.

Our predictions concerning wood litter production can also be compared with empirical data. Dewit and Reid (1992, fig. 3), reproduced here in figure $9 a$, record the age distribution of branches shed from a balsam poplar ( $P$. balsamifera) during one autumn. Their recorded age distribution has the same general shape as our model-generated age distributions shown in figure A3, apart from the low number of abscised 10-year-old branches, which Dewit and Reid speculate was caused by a transplantation of the tree with a large mechanical tree spade that took place in 1977, 10 years prior to their measurements. In particular, there is a minimum age below which very few branches are shed, a peak at a fairly low age, and an extended tail toward older branches.

While the distributions share the same qualitative features, our model-generated distributions in figure A3 have thinner tails and sharper concentration of discarded branches at the age at which branches first exceed their branch-carrying capacity. To see whether the thicker tails can be explained by a gradual transition to branch thinning, rather than the abrupt onset we have assumed thus far, we replace the minimum function assumed in equation (4) with a smooth approximation and find, as we show in figure $9 b$, that the thicker tail can also be captured within our modeling framework.

\section{Discussion}

As trees grow in height, they lose branches to extrinsic factors such as wind breakage, but many tree species also cease to support branches that eventually die or actively abscise branches (Schaffner and Tyler 1901; Mäkinen 1999; Mäkinen and Colin 1999; Heräjärvi 2001; Mäkinen and Hein 2006). The wood litter produced can be substantial; the pine tree in figure $2 a$ must have lost every branch that once existed at heights below its first branch, and the eucalyptus tree in figure $2 b$ must have lost a comparable number of branches. Wood litter is important in the carbon cycle and as a factor influencing both the growth and branching architecture of trees. We have introduced and explored a simple model of tree growth in which branches are thinned whenever they exceed a branch carrying capacity. By assuming a branch carrying capacity, we were able to derive approximate analytical predictions for tree architecture at any point in time and for tree wood litter production over 

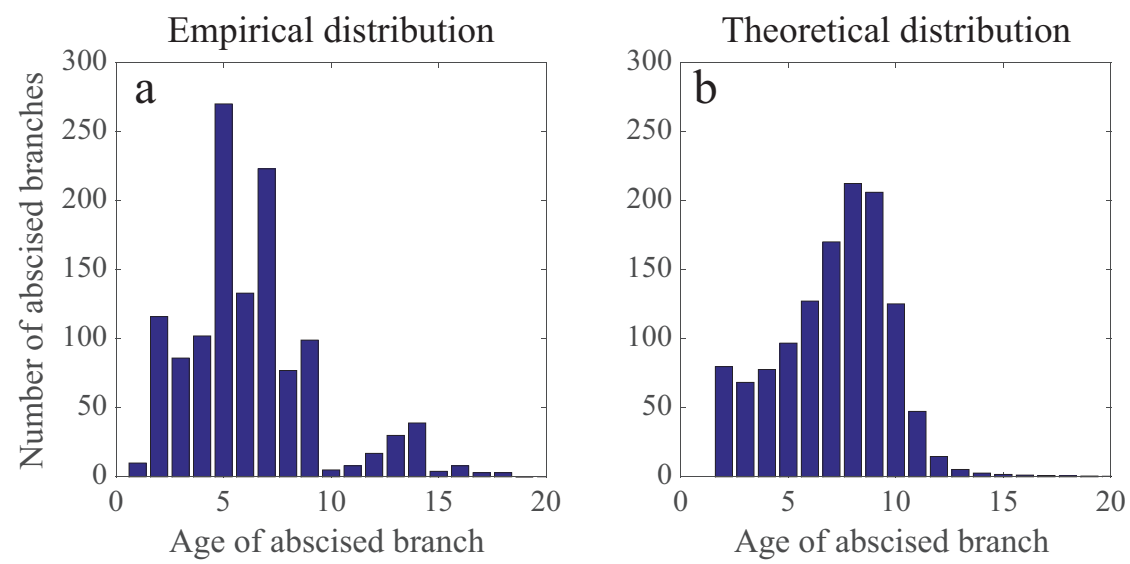

Figure 9: Our prediction for the age distribution of discarded branches compares favorably with empirical measurements. $a$, Data from Dewit and Reid (1992) on the age distribution of abscised branches between October and December 1987 in one specimen of balsam poplar (Populus balsamifera). b, Model-generated age distribution of abscised branches during one growth cycle, assuming that all discarded branches are abscised. The distribution is calculated from equation (8) using a smooth approximation of the minimum function to represent a gradual onset of branch thinning. Specifically, we used $\left(\left(x^{q}+y^{q}\right) / 2\right)^{1 / q}$ with $q=-2$ as a smooth approximation of $\min (x, y)$ in equation $(4)$. Parameters are $\beta=10, d=3, \mu=2.5$, and $n=100$. See table 1 for variable and parameter definitions.

time, both expressed as the total biomass lost and as a size mixture of dead and abscised branches.

With our model being a simplified representation of tree growth, to what extent do our predictions apply more generally? Using individual-based simulations we found that our results remain robust under stochastic branching patterns and branch thinning that, when the branch carrying capacity has been exceeded, discards subbranches closer to their carrying capacity with higher probability. Most tree species, however, avoid the untamed exponential branching pattern assumed here by differentiating rules for separate classes of branches, often denoted apparent branching order (figure A5; see also Hallé et al. 1978; de Reffye et al. 1988; Barthélémy and Caraglio 2007). The phenomenological carrying capacity assumed here is also in reality realized through other factors, such as light or nutrient limitation. Existing functional-structural plant models (see, e.g., Cournède et al. 2008; Vos et al. 2010) that model detailed aspects of tree growth could potentially be used to study how more realistic assumptions on tree growth influence the predicted wood litter production (see Eschenbach 2005 for a step in this direction).

The ideal test of our predictions is to confront them with empirical data. We have taken a first step in this direction by comparing our predictions for the number of tips on a branch over time with Wilson's (1966) observations of red maple and the age distribution of wood litter produced with Dewit and Reid's (1992) observations of balsam poplar. In both cases, we found qualitative agreement that increases the likelihood that our conclusions may extend beyond the simplified growth model in which they were derived.
In the appendix section Empirical Measurement of Model Parameters, we discuss how the model parameters may be inferred from field observations. If data on wood litter production can also be obtained, the predicted wood litter production from our model can be compared with the actual values at both a quantitative and a qualitative level. It might also be possible to compare directly to architectural data, such as our prediction that the distances between consecutive branching points as a branch is traversed from tip to stem should (except in the tree periphery) on average increase with a factor $2^{1 / d}$, in which $d$ is one of the measured model parameters. Advances in remote sensing may even allow automated data collection (see Lindberg and Holmgren 2017 for a recent review). A follow-up study that considers the consequences of more detailed representations of plant growth or that interfaces with empirical data, for example as outlined above, would be valuable; such an effort would be likely to yield important insights independent of whether it supports the predictions and conceptual ideas presented here.

Our model builds on the idea that trees produce more branches than they can support and that some of these branches must therefore be lost. A related idea is at the heart of a strand of computer algorithms for generating tree forms, in which branches compete to colonize different areas of available space (Ulam 1962; Cohen 1967; Prusinkiewicz and de Reuille 2010). While these algorithms build on the realization that physical space is limited, they are in practice quite different from our modeling framework. A more closely related body of work is the West-BrownEnquist theory for trees (West et al. 1999; see also Enquist 
2002; Price et al. 2010; Savage et al. 2010; Smith et al. 2014), which combines a structural-mechanical model of branches and a hydraulic model of the vascular system. Branches are assumed to be self-similar in the sense that subbranches are architecturally scaled-down versions of the full branch, with a cutoff at the scale of petioles. Starting with this static view of plants, the theory predicts rates of fluid flow and biomass production in relation to plant size. By contrast, we do not primarily consider tree morphology at a point in time but rather how it develops over time through the growth and loss of branches. Another model capable of predicting wood litter has been developed by Mäkelä (1997). By assuming that carbon is lost through death of the lowest branches as the height over ground of the tree crown increases, the total rate of wood litter production is determined. Importantly, however, this model does not consider branching structure and therefore cannot be used to study the size distribution of branches or the consequences of self-pruning for the emergent branching architecture.

In this article, we have emphasized that the large-scale, self-similar branching structure of trees emerges as a consequence of branch-thinning during ontogeny. Using a simplified model of tree growth, we have shown how to derive approximate analytical predictions for tree architecture at any point in time and for tree wood litter production over time, both expressed as the total biomass lost and as the size mixture of dead and abscised branches. Such predictions have value that extends beyond increased understanding of plant ecology; they can potentially be used to improve models of the carbon cycle and also to improve the dynamic global vegetation models that are used to study climate change. Further work will tell how well our model predictions match data and improve on the model given here by allowing more realistic growth patterns.

\section{Acknowledgments}

This work was primarily supported by a Young Researcher Award from Umeå University to Å.B. Additional support from the Australian Research Council, the Swedish Research Council, and the Swedish Research Council Formas are gratefully acknowledged. We thank Ben Bolker and three anonymous reviewers for valuable comments that helped to greatly improve this article. The photos in figure $2 a$ and $2 b$ are shot by Pavel Šinkyř́k and D.F., respectively.

\section{Literature Cited}

Barthélémy, D., and Y. Caraglio. 2007. Plant architecture: a dynamic, multilevel and comprehensive approach to plant form, structure and ontogeny. Annals of Botany 99:375-407.

Borchert, R., and N. A. Slade. 1981. Bifurcation ratios and the adaptive geometry of trees. Botanical Gazette 142:394-401.
Cohen, D. 1967. Computer simulation of biological pattern generation processes. Nature 216:246-248.

Cournède, P.-H., A. Mathieu, F. Houllier, D. Barthélémy, and P. de Reffye. 2008. Computing competition for light in the GreenLab model of plant growth: a contribution to the study of the effects of density on resource acquisition and architectural development. Annals of Botanv 101:1207-1219.

de Reffye, P., C. Edelin, J. Françon, M. Jaeger, and C. Puech. 1988. Plant models faithful to botanical structure and development. Pages 151-158 in Proceedings of the 15th Annual Conference on Computer Graphics and Interactive Techniques (SIGGRAPH '88). Vol. 22. Association for Computing Machinery, New York.

Dewit, L., and D. M. Reid. 1992. Branch abscission in balsam poplar (Populus balsamifera): characterization of the phenomenon and the influence of wind. International Journal of Plant Sciences 153:556-564.

Enquist, B. J. 2002. Universal scaling in tree and vascular plant allometry: toward a general quantitative theory linking plant form and function from cells to ecosystems. Tree Physiology 22:10451064 .

Enquist, B. J., G. B. West, E. L. Charnov, and J. H. Brown. 1999. Allometric scaling of production and life-history variation in vascular plants. Nature 401:907-911.

Eschenbach, C. 2005. Emergent properties modelled with the functional structural tree growth model ALMIS: computer experiments on resource gain and use. Ecological Modelling 186:470-488.

Ferraro, P., C. Godin, and P. Prusinkiewicz. 2005. Toward a quantification of self-similarity in plants. Fractals 13:91-109.

Ford, E. D. 2014. The dynamic relationship between plant architecture and competition. Frontiers in Plant Science 5:275.

Godin, C., and P. Ferraro. 2010. Quantifying the degree of selfnestedness of trees: application to the structural analysis of plants. IEEE/ACM Transactions on Computational Biology and Bioinformatics 7:688-703.

Hallé, F., R. A. Oldeman, and P. B. Tomlinson. 1978. Tropical trees and forests: an architectural analysis. Springer, Berlin.

Hellström, L. 2018. lars-hellstrom/branch-thin-archival: branchthinning simulation source code and data, for American Naturalist paper. Zenodo. https://zenodo.org/record/1160465.

Heräjärvi, H. 2001. Technical properties of mature birch (Betula pendula and B. pubescens) for saw milling. Silva Fennica 4:469-485.

Lindberg, E., and J. Holmgren. 2017. Individual tree crown methods for 3D data from remote sensing. Current Forestry Reports 3:19-31.

Lindenmayer, A. 1968. Mathematical models for cellular interactions in development. I. Filaments with one-sided inputs. Journal of Theoretical Biology 18:280-299.

Mäkelä, A. 1997. A carbon balance model of growth and self-pruning in trees based on structural relationships. Forest Science 43:7-24.

Mäkinen, H. 1999. Growth, suppression, death, and self-pruning of branches of Scots pine in southern and central Finland. Canadian Journal of Forest Research 29:585-594.

Mäkinen, H., and F. Colin. 1999. Predicting the number, death, and self-pruning of branches in Scots pine. Canadian Journal of Forest Research 29:1225-1236.

Mäkinen, H., and S. Hein. 2006. Effect of wide spacing on increment and branch properties of young Norway spruce. European Journal of Forest Research 125:239-248.

McMahon, T. A., and R. E. Kronauer. 1976. Tree structures: deducing the principle of mechanical design. Journal of Theoretical Biology 59:443-466. 
Price, C. A., J. F. Gilooly, A. P. Allen, J. S. Weitz, and K. J. Niklas. 2010. The metabolic theory of ecology: prospects and challenges for plant biology. New Phvtologist 188:696-710.

Prusinkiewicz, P. 2004. Self-similarity in plants: integrating mathematical and biological perspectives. Pages 103-118 in M. M. Novak, ed. Thinking in patterns: fractals and related phenomena in nature. World Scientific, Singapore.

Prusinkiewicz, P., and P. B. de Reuille. 2010. Constraints of space in plant development. Journal of Experimental Botany 61:21172129.

Prusinkiewicz, P., Y. Erasmus, B. Lane, L. D. Harder, and E. Coen. 2007. Evolution and development of inflorescence architectures. Science 316:1452-1456.

Prusinkiewicz, P., and A. Lindenmayer. 1990. The algorithmic beauty of plants. Springer, New York.

Savage, V. M., L. P. Bentley, B. J. Enquist, J. S. Sperry, D. D. Smith, P. B. Reich, and E. I. von Allmen. 2010. Hydraulic trade-offs and space filling enable better predictions of vascular structure and function in plants. Proceedings of the National Academv of Sciences of the USA 107:22722-22727.

Schaffner, J. H., and F. J. Tyler. 1901. Notes on the self-pruning of trees. Ohio State University Naturalist 1:29-32.
Smith, D. D., J. S. Sperry, B. J. Enquist, V. M. Savage, K. A. McCulloh, and L. P. Bentley. 2014. Deviation from symmetrically self-similar branching in trees predicts altered hydraulics, mechanics, light interception and metabolic scaling. New Phytologist 201:217-229.

Ulam, S. 1962. On some mathematical properties connected with patterns of growth of figures. Pages 215-224 in R. E. Bellman, ed. Proceedings of Symposia on Applied Mathematics. Vol. 14. Mathematical problems in the biological sciences. American Mathematical Society, Providence, RI.

Vos, J., J. B. Evers, G. H. Buck-Sorlin, B. Andrieu, M. Chelle, and P. H. B. De Visser. 2010. Functional-structural plant modelling: a new versatile tool in crop science. Journal of Experimental Botany 61:2101-2115.

West, G. B., J. H. Brown, and B. J. Enquist. 1997. A general model for the origin of allometric scaling laws in biology. Science 276:122-126. 1999. A general model for the structure and allometry of plant vascular systems. Nature 400:664-667.

Wilson, B. F. 1966. Development of the shoot system of Acer rubrum L. Harvard University Press, Petersham, MA.

Associate Editor: Benjamin M. Bolker Editor: Alice A. Winn

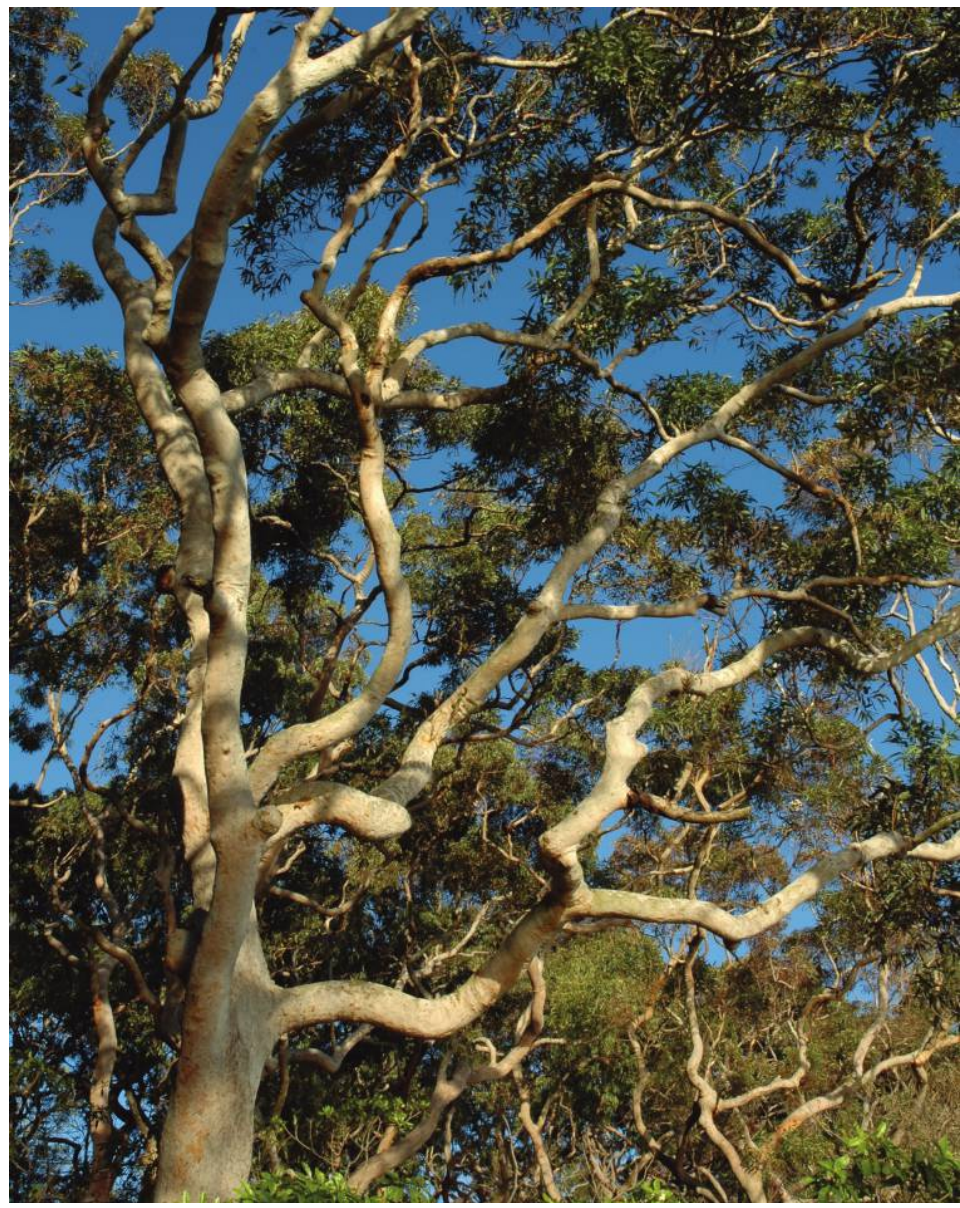

This tree, Angophora costata, exhibits an emergent, self-similar, branching structure that may have arisen as a result of carrying capacity constraints on the number of branch tips that can be maintained. Photo credit: Daniel Falster. 